\title{
Existence of solution and Hyers-Ulam stability for a coupled system of fractional differential equations with p-Laplacian operator
}

\author{
Hasib Khan ${ }^{\mathrm{a}, \mathrm{b}}$, Yongjin Lic,*, Hongguang Sun ${ }^{\mathrm{a}}$, Aziz Khan ${ }^{\mathrm{d}}$ \\ a State Key Laboratory of Hydrology-Water Resources and Hydraulic Engineering, Hohai University, 210098, Nanjing, P. R. China. \\ ${ }^{b}$ Shaheed Benazir Bhutto University Sheringal, Dir Upper, 18000, Khyber Pakhtunkhwa, Pakistan. \\ ${ }^{c}$ Department of Mathematics, Sun Yat-sen University, 510275, Guangzhou, P. R. China. \\ ${ }^{d}$ Department of Mathematics, University of Peshawar, 25000, Khyber Pakhtunkhwa, Pakistan.
}

Communicated by J. Brzdek

\begin{abstract}
Models with p-Laplacian operator are common in different scientific fields including; plasma physics, chemical reactions design, physics, biophysics, and many others. In this paper, we investigate existence and uniqueness of solution and Hyers-Ulam stability for a coupled system of fractional differential equations with p-Laplacian operator. The Hyers-Ulam stability means that a differential equation has a close exact solution which is generated by the approximate solution of the differential equation and the error in the approximation can be estimated. We use topological degree method and provide an expressive example as an application of the work. (C)2017 All rights reserved.
\end{abstract}

Keywords: Existence and uniqueness of solution, Hyers-Ulam stability, topological degree method, p-Laplacian operator. 2010 MSC: $47 \mathrm{H} 10,54 \mathrm{H} 25$.

\section{Introduction and preliminaries}

The real world physical phenomena described by mathematical models of fractional differential equations (FDEs) are more useful and realistic in memory as compared to the models of integer order differential equations. In last two decades, scientists have shown a great attention to the applications of FDEs in various disciplines like signals, fluid dynamics, biology, hydrodynamics, viscoelastic theory, image processing, control theory, computer networking, and many others [2, 8, 15-17, 30, 31]. Existence of solutions for FDEs is one of the most interested research areas. The researchers use different methods for the study of existence and uniqueness of solution for the different classes of FDEs [4, 11, 26-29].

For instance, Baleanu and Mustafa [4] studied global existence of solutions to a class of FDEs and provided applications of their results. Baleanu et al. [5] obtained some results for the existence of solution for a superlinear FDE and established the applications of their results. Baleanu et al. [3] proved some results for the existence of solution for a nonlinear FDEs on partially ordered Banach spaces and provided

\footnotetext{
${ }^{*}$ Corresponding author

Email addresses: hasibkhan13@yahoo.com (Hasib Khan), stslyj@mail.sysu.edu.cn (Yongjin Li), shg@hhu.edu.cn (Hongguang Sun), azizkhan927@yahoo.com (Aziz Khan) 
applications of their results. Baleanu et al. [6] studied that under certain assumptions that the solutions of FDEs are eventually large and eventually small. Mahmudov and Unul [20] studied a fractional differential equation of order $\alpha \in(2,3]$ with integral conditions, an impulsive fractional differential equation [22] and fractional differential equation with p-Laplacian operator [21], for the existence of solutions. Hu et al. [13] studied a coupled system of fractional differential equations with nonlinear $p$-Laplacian operator at resonance. Kuman et al. [19] studied stability and existence results for a nonlinear FDE.

Here, we highlight the contributions of some well-known scientists which have provided a base to our present work. For instance, Prasad [26] studied existence of positive solutions for the coupled system fractional order boundary value problem with $p$-Laplacian operator of the kind

$$
\begin{aligned}
& \mathfrak{D}_{0^{+}}^{\beta_{1}}\left(\phi_{\mathfrak{p}}\left(\mathfrak{D}_{0^{+}}^{\alpha_{1}} \mathfrak{v}(\mathrm{t})\right)\right)=\mathrm{f}_{1}(\mathrm{t}, \boldsymbol{v}(\mathrm{t}), z(\mathrm{t})), \quad \mathfrak{D}_{0^{+}}^{\beta_{2}}\left(\phi_{\mathfrak{p}}\left(\mathfrak{D}_{0^{+}}^{\alpha_{2}} \mathfrak{v}(\mathrm{t})\right)\right)=\mathrm{f}_{2}(\mathrm{t}, \boldsymbol{v}(\mathrm{t}), z(\mathrm{t})), \\
& v(0)=\mathfrak{D}_{0^{+}}^{\mathfrak{q}_{1}} \mathfrak{v}(1)=0, \quad \gamma \boldsymbol{v}(1)+\delta \mathfrak{D}_{0^{+}}^{\mathrm{q}_{2}} \boldsymbol{v}(1)=0, \\
& v(0)=\mathfrak{D}_{0^{+}}^{\mathfrak{q}_{1}} z(0)=0, \quad \gamma v(1)+\delta \mathfrak{D}_{0^{+}}^{\mathfrak{q}_{2}} z(1)=0, \\
& \mathfrak{D}_{0^{+}}^{\alpha_{1}} \mathfrak{v}(0)=\mathfrak{D}_{0^{+}}^{\alpha_{1}} \mathfrak{v}(1)=0 \\
& \mathfrak{D}_{0^{+}}^{\alpha_{2}} z(0)=\mathfrak{D}_{0^{+}}^{\alpha_{2}} z(1)=0,
\end{aligned}
$$

where $t \in(0,1), 2<\alpha_{i} \leqslant 3,1<\beta_{i}, q_{i} \leqslant 2, \gamma, \delta$ are real positive numbers, $f_{i}:[0,1] \times \mathbb{R}^{2} \rightarrow \mathbb{R}^{2}$ are continuous function, and the fractional derivatives $\mathfrak{D}_{0^{+}}^{\alpha_{i}}, \mathfrak{D}_{0^{+}}^{\beta_{i}}, \mathfrak{D}_{0^{+}}^{q_{i}}$ are in the Riemann-Liouville sense. Cheng et al. [9] studied the resonance and coupled problem FDEs of $p$-Laplacian for existence of solutions, by using degree theory

$$
\begin{aligned}
\mathfrak{D}_{0^{+}}^{\gamma}\left(\phi_{\mathfrak{p}}\left(\mathfrak{D}_{0^{+}}^{\alpha} \mathfrak{v}(\mathrm{t})\right)\right) & =f_{1}(t, z(t)), \mathfrak{D}_{0^{+}}^{\gamma}\left(\phi_{\mathfrak{p}}\left(\mathfrak{D}_{0^{+}}^{\beta} z(t)\right)\right)=f_{2}(t, v(t)), \\
\mathfrak{D}_{0^{+}}^{\alpha} \mathfrak{v}(t) & =\mathfrak{D}_{0^{+}}^{\alpha} \mathfrak{v}(1)=\mathfrak{D}_{0^{+}}^{\beta} z(0)=\mathfrak{D}_{0^{+}}^{\beta} z(1)=0,
\end{aligned}
$$

where $f, g:[0,1] \times \mathbb{R} \rightarrow \mathbb{R}, \mathrm{t} \in[0,1], 0<\gamma \leqslant 1, \mathrm{n}-1<\alpha, \beta \leqslant \mathrm{n}$ are continuous functions. $\mathfrak{D}_{0^{+}}^{\alpha}, \mathfrak{D}_{0^{+}}^{\beta}$ and $\mathfrak{D}_{0^{+}}^{\gamma}$ represent Caputo fractional derivatives. Urs [32] derived the Hyers-Ulam stability for the following coupled system

$$
\begin{aligned}
u^{\prime \prime}(t)-\theta_{1}(t, v(t)) & =\theta_{2}(t, z(t)), \quad v^{\prime \prime}(t)-\theta_{1}(t, z(t))=\theta_{2}(t, v(t)), \\
\left.v(t)\right|_{t=0} & =v(t)_{t=T},\left.\quad z(t)\right|_{t=0}=z(t)_{t=T} .
\end{aligned}
$$

Brzdek et al. [7] presented a survy on Ulam stability. Ali et al. [1] studied Hyers-Ulam stability by the help of topological degree theory for existence and uniqueness of solution for FDEs

$$
\begin{gathered}
\mathfrak{D}_{0_{+}}^{p} v(t)=\theta_{1}(t, z(t)), \mathfrak{D}_{0_{+}}^{q} v(t)=\theta_{2}(t, v(t)), \quad t \in J=[0,1], \\
v(0)=0, v(1)=I_{T}^{\gamma} g(u)=\frac{1}{\Gamma \gamma} \int_{0}^{T}(T-s)^{\gamma-1} g(v(s)) d s, \\
z(0)=0, z(1)=I_{T}^{\delta} h(v)=\frac{1}{\Gamma \delta} \int_{0}^{T}(T-s)^{\delta-1} h(z(s)) d s,
\end{gathered}
$$

where $p, q, \delta, \gamma \in(1,2]$ and $\mathfrak{D}_{0_{+}}^{p}, \mathfrak{D}_{0_{+}}^{q}$ denote Caputo derivatives.

Inspired from the above work, we investigate existence and uniqueness of solution and Hyers-Ulam stability for the following coupled system of FDEs with fractional differential and integral initial and boundary conditions with p-Laplacian operator

$$
\begin{aligned}
& \mathfrak{D}_{0_{+}}^{\beta_{1}} \phi_{\mathfrak{p}}\left(\mathfrak{D}_{0_{+}}^{\alpha_{1}} \mathfrak{v}(\mathrm{t})\right)=-\psi_{1}(\mathrm{t}, z(\mathrm{t})), \quad \mathfrak{D}_{0_{+}}^{\beta_{2}} \phi_{\mathfrak{p}}\left(\mathfrak{D}_{0_{+}}^{\alpha_{2}} z(t)\right)=-\psi_{2}(\mathrm{t}, \mathfrak{v}(\mathrm{t})),
\end{aligned}
$$

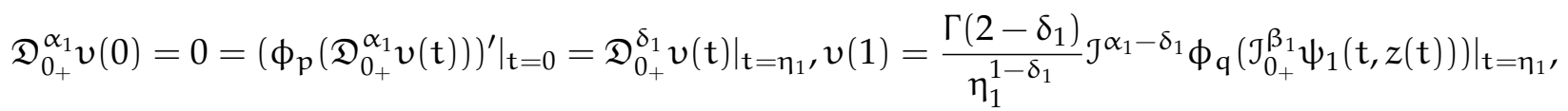

$$
\begin{aligned}
& \mathfrak{D}_{0_{+}}^{\alpha_{2}} z(0)=0=\left.\left(\phi_{\mathfrak{p}}\left(\mathfrak{D}_{0_{+}}^{\alpha_{2}} z(\mathrm{t})\right)\right)^{\prime}\right|_{\mathfrak{t}=0}=\left.\mathfrak{D}_{0_{+}}^{\delta_{2}} z(\mathrm{t})\right|_{\mathfrak{t}=\eta_{2}}, v(1)=\left.\frac{\Gamma\left(2-\delta_{2}\right)}{\eta_{2}^{1-\delta_{2}}} \mathfrak{J}^{\alpha_{2}-\delta_{2}} \phi_{\mathrm{q}}\left(\mathcal{J}_{0_{+}}^{\beta_{2}} \psi_{2}(\mathrm{t}, \mathfrak{v}(\mathrm{t}))\right)\right|_{\mathrm{t}=\eta_{2}},
\end{aligned}
$$

where $t \in[0,1], \eta_{i}, \delta_{i} \in(0,1), \alpha_{i}, \beta_{i} \in(1,2]$, for $i=1,2$. The nonlinear $p$-Laplacian operator satisfies $\phi_{p}(s)=|s|^{p-2} s, p>1, \phi_{p}^{-1}=\phi_{q}, \frac{1}{p}+\frac{1}{q}=1$, and $\mathfrak{D}_{0_{+}}^{\alpha_{i}}, \mathfrak{D}_{0_{+}}^{\beta_{i}}, \mathfrak{D}_{0_{+}}^{\delta_{i}}$ for $i=1,2$ are in the sense of Caputo fractional derivative. Indeed our problem (1.1) is more general than the one considered in the references. We give some basic definitions and results from the available literature $[8,12,18,23-25]$. 
Definition 1.1. The fractional integral of order $\alpha>0$ of a function $\psi:(0,+\infty) \rightarrow \mathbb{R}$ is given by

$$
\mathcal{J}_{0+}^{\alpha} \psi(t)=\frac{1}{\Gamma(\alpha)} \int_{0}^{t}(t-s)^{\alpha-1} \psi(s) d s
$$

provided that the integral on right side is pointwise defined on the interval $(0,+\infty)$, where

$$
\Gamma(\alpha)=\int_{0}^{+\infty} e^{-s} s^{\alpha-1} \mathrm{ds}
$$

Definition 1.2. The Caputo fractional derivative of order $\alpha>0$ for a continuous function $\psi(t):(0,+\infty) \rightarrow$ $\mathbb{R}$ is defined by

$$
\mathfrak{D}_{0^{+}}^{\alpha} \psi(t)=\frac{1}{\Gamma(k-\alpha)} \int_{0}^{t}(t-s)^{k-\alpha-1} \psi^{(k)}(s) d s
$$

for $k=[\alpha]+1$, where $[\alpha]$ is used for the integer part of $\alpha$, provided that the integral on right side is pointwise defined on $(0,+\infty)$.

Lemma 1.3 ([18]). Let $\alpha \in(n-1, n], \psi \in A C^{n-1}$, then

$$
\mathcal{J}_{0_{+}}^{\alpha} \mathfrak{D}_{0_{+}}^{\alpha} \psi(t)=\psi(t)+c_{0}+c_{1} t+c_{2} t^{2}+\cdots+c_{n-1} t^{n-1}
$$

for $c_{i} \in \mathbb{R}$ for $i=0,1,2, \ldots, n-1$.

Consider the space of real-valued continuous functions $\mathcal{U}=\mathrm{C}([0,1], \mathbb{R})$ with the topological norm defined by $\|v\|=\sup \{|v(t)|: 0 \leqslant t \leqslant 1\}$ for $v \in \mathcal{U}$. The product space $\omega=\mathcal{U} \times \mathcal{U}$ with the norm $\|(v, z)\|=\|v\|+\|z\|$ is also a Banach space. We represent the class of all bounded mappings in $\omega$ by $\mathcal{S}$.

Definition 1.4. The mapping $\xi: \mathcal{S} \rightarrow(0, \infty)$ for Kuratowski measure of non-compactness is defined as:

$$
\xi(\wp)=\inf \{d>0: \wp \quad \text { the finite cover for sets of diameter } \leqslant d\},
$$

where $\wp \in \mathcal{S}$.

Definition 1.5. Assume that $\mathcal{T}: \vartheta \rightarrow \mathcal{U}$ is a bounded and continuous mapping $\vartheta \subset \mathcal{U}$. Then $\mathcal{T}$ is a $\xi$-Lipschitz where $\zeta \geqslant 0$ such that

$$
\xi(\mathcal{T}(\wp)) \leqslant \zeta \xi(\wp) \quad \text { for all bounded } \wp \subset \vartheta .
$$

Then $\mathcal{T}$ is called strict $\xi$-contraction if $\zeta<1$.

Definition 1.6. The function $\mathcal{T}$ is $\xi$-condensing if

$$
\xi(\mathcal{T}(\wp))<\xi(\wp) \text { for all bounded } \wp \subset \vartheta \text { such that } \xi(\wp)>0 .
$$

Therefore $\xi(\mathcal{T}(\wp)) \geqslant \xi(\wp)$ yields $\xi(\wp)=0$.

Further we have $\mathcal{T}: \vartheta \rightarrow \mathcal{U}$ is Lipschitz for $\zeta>0$, such that

$$
\|\mathcal{T}(v)-\mathcal{T}(\bar{v})\| \leqslant \zeta\|v-\bar{v}\| \quad \text { for all } v, \bar{v} \in \vartheta
$$

For the condition $\zeta<1, \mathcal{T}$ is a strict contraction.

Proposition 1.7. The mapping $\mathcal{T}$ is $\xi$-Lipschitz with constant $\zeta=0$ if and only if $\mathcal{T}: \vartheta \rightarrow U$ is compact.

Proposition 1.8. The operator $\mathcal{T}$ is $\xi$-Lipschitz with constant $\zeta$ if and only if $\mathcal{T}: \vartheta \rightarrow \mathcal{U}$ is Lipschitz with constant $\zeta$. 
Theorem 1.9 ([14]). Let $\mathcal{T}: \mathcal{U} \rightarrow \mathcal{U}$ be a $\xi$-codensing and

$$
\mathcal{H}=\{z \in \mathcal{U} \text { : there exists } 0 \leqslant \lambda \leqslant 1 \text { such that } z=\lambda \mathcal{T} z \text {. }
$$

If $\mathcal{H}$ is a bounded set in $\mathcal{U}$, so there exists $a>0$, such that $\mathcal{H} \subset \wp_{a}(0)$, then

$$
\operatorname{deg}\left(\mathrm{I}-\lambda \mathcal{G}, \wp_{\mathrm{r}}(0), 0\right)=1 \text { for all } \lambda \in[0,1] .
$$

Consequently, $\mathcal{T}$ has at least one fixed point and the set of the fixed points of $\mathcal{T}$ lies in $\wp_{\mathrm{a}}(0)$.

The next Lemma 1.10 has an important roll in this paper.

Lemma 1.10 ([28]). Let $\phi_{p}$ be a $p$-Laplacian operator. Then

(1) if $1<p \leqslant 2, \ell_{1} \ell_{2}>0$, and $\left|\ell_{1}\right|,\left|\ell_{2}\right| \geqslant m>0$, then

$$
\left|\phi_{p}\left(\ell_{1}\right)-\phi_{p}\left(\ell_{2}\right)\right| \leqslant(p-1) m^{p-2}\left|\ell_{1}-\ell_{2}\right| ;
$$

(2) if $\mathrm{p}>2$, and $\left|\ell_{1}\right|,\left|\ell_{2}\right| \leqslant M$, then

$$
\left|\phi_{p}\left(\ell_{1}\right)-\phi_{p}\left(\ell_{2}\right)\right| \leqslant(p-1) M^{p-2}\left|\ell_{1}-\ell_{2}\right| .
$$

We use the following assumptions in the proofs of our main results.

$\left(\mathrm{A}_{1}\right)$ The functions $\psi_{1}, \psi_{2}$ satisfy the following growth conditions for constants $a, b, \mathcal{M}_{1}^{*}, \mathcal{M}_{2}^{*}, p, p \in(0,1]$.

$$
\left|\psi_{1}(t, z)\right| \leqslant a|z|^{p}+\mathcal{M}_{1}^{*}, \quad\left|\psi_{2}(t, v)\right| \leqslant b|v|^{p}+\mathcal{M}_{2}^{*} .
$$

$\left(A_{2}\right)$ There exist real-valued constants $L_{\psi_{1}}, L_{\psi_{2}}$, and for all $x, y, \sigma, \sigma^{*} \in \mathcal{U}$,

$$
\left|\psi_{1}(t, y)-\psi_{1}(t, \sigma)\right| \leqslant L_{\psi_{1}}|y-\sigma|, \quad\left|\psi_{2}(t, x)-\psi_{1}\left(t, \sigma^{*}\right)\right| \leqslant L_{\psi_{1}}\left|x-\sigma^{*}\right|
$$

The Hyers-Ulam stability for differential equations has been considered by many scientists. For example, Gâvrută et al. [10] investigated the Hyers-Ulam stability for following second order differential equation

$$
y^{\prime \prime}+\beta(x) y=0,
$$

with the boundary conditions

$$
y(a)=y^{\prime}(b)=0,
$$

or initial condition

$$
y(a)=y^{\prime}(a)=0,
$$

where $y \in C^{2}[a, b], \beta(x) \in C[a, b],-\infty<a<b<+\infty$. They gave the following definition for the Hyers-Ulam stability of (1.2).

Definition 1.11 ([10]). The equation (1.2) has Hyers-Ulam stability with boundary conditions (1.3), if there exists a positive constant $\mathcal{D}$ satisfying: for every $\epsilon>0, y \in C^{2}[a, b]$, if

$$
\left|y^{\prime \prime}+\beta(t) y\right| \leqslant \epsilon
$$

and $y(a)=0=y(b)$, then there exists some $u \in C^{2}[a, b]$ satisfying

$$
u^{\prime \prime}+\beta(t) u=0
$$

and $u(a)=0=u(b)$, such that $|y(t)-u(t)|<\mathcal{D} \epsilon$.

For the existence and uniqueness of solution, Hyers-Ulam stability, application, and conclusion of the paper, we have further four sections. In Section 2, we give the equivalent integral form of the problem (1.1) by the help of Green's functions and existence and uniqueness of solution for the problem (1.1) will be discussed. The Hyers-Ulam stability of the system (1.1) has been proved in Section 3. In Section 4, an illustrative example is given for the application of our results. The whole paper is then summarized in Section 5 . 


\section{Existence and uniqueness}

Theorem 2.1. Let $\psi(t) \in C[0,1]$, then the following FDE with fractional order differential and integral initial and boundary conditions

$$
\begin{aligned}
& \mathfrak{D}_{0_{+}}^{\beta_{1}} \phi_{\mathfrak{p}}\left(\mathfrak{D}_{0_{+}}^{\alpha_{1}} \mathfrak{v}(\mathrm{t})\right)=-\psi(\mathrm{t}), \\
& \mathfrak{D}_{0_{+}}^{\alpha_{1}} \mathfrak{v}(0)=0=\left.\left(\phi_{\mathfrak{p}}\left(\mathfrak{D}_{0_{+}}^{\alpha_{1}} \mathfrak{v}(\mathrm{t})\right)\right)^{\prime}\right|_{\mathrm{t}=0}=\left.\mathfrak{D}_{0_{+}}^{\delta_{1}} \mathfrak{v}(\mathrm{t})\right|_{\mathrm{t}=\eta_{1}}, \quad v(1)=\left.\frac{\Gamma\left(2-\delta_{1}\right)}{\eta_{1}^{1-\delta_{1}}} \mathfrak{J}_{0_{+}}^{\alpha_{1}-\delta_{1}} \phi_{\mathrm{q}}\left(\mathcal{J}_{0_{+}}^{\beta_{1}} \psi(\mathrm{t})\right)\right|_{\mathrm{t}=\eta_{1}}
\end{aligned}
$$

has a solution

$$
v(t)=\int_{0}^{1} \mathcal{G}_{\alpha_{1}}(t, s) \phi_{q}\left(\mathcal{J}_{0_{+}}^{\beta_{1}} \psi(\tau)\right) d s,
$$

where $\mathcal{G}_{\alpha_{1}}(t, s)$ is a Green function defined by

$$
\mathcal{G}_{\alpha_{1}}(t, s)= \begin{cases}\frac{-(\mathrm{t}-\mathrm{s})^{\alpha_{1}-1}+(1-s)^{\alpha_{1}-1}}{\Gamma\left(\alpha_{1}\right)}+\frac{\mathrm{t} \Gamma\left(2-\delta_{1}\right)}{\eta_{1}^{1-\delta_{1}} \Gamma\left(\alpha_{1}-\delta_{1}\right)}\left(\eta_{1}-s\right)^{\alpha_{1}-\delta_{1}-1}, & s \leqslant \eta_{1} \leqslant t \leqslant 1, \\ -\frac{(t-s)^{\alpha_{1}-1}}{\Gamma \alpha_{1}}+\frac{(1-s)^{\alpha_{1}-1}}{\Gamma\left(\alpha_{1}\right)}, & \eta_{1} \leqslant s \leqslant t \leqslant 1 \\ \frac{(1-s)^{\alpha_{1}-1}}{\Gamma\left(\alpha_{1}\right)}, & \eta_{1} \leqslant t \leqslant s \leqslant 1 .\end{cases}
$$

Proof. Applying the operator $\mathcal{J}_{0_{+}}^{\beta}$ on (2.1) and using Lemma 1.3, we have

$$
\phi_{p}\left(\mathfrak{D}_{0_{+}}^{\alpha_{1}} v(t)\right)=-\mathcal{J}_{0_{+}}^{\beta_{1}} \psi(t)+c_{0}+c_{1} t .
$$

By using conditions $\left.\left(\phi_{\mathfrak{p}}\left(\mathfrak{D}_{0_{+}}^{\alpha_{1}} \mathfrak{v}(\mathrm{t})\right)\right)^{\prime}\right|_{\mathfrak{t}=0}=0=\mathfrak{D}_{0_{+}}^{\alpha_{1}} \mathfrak{v}(0)$, we get $\mathfrak{c}_{0}=\mathfrak{c}_{1}=0$. From (2.2) we have

$$
v(t)=-\mathcal{J}^{\alpha_{1}}\left(\phi_{\mathrm{q}}\left(\mathcal{J}_{0_{+}}^{\beta_{1}} \psi(t)\right)\right)+c_{2}+c_{3} t .
$$

By the help of condition $\mathfrak{D}_{0_{+}}^{\delta_{1}} v\left(\eta_{1}\right)=0$ in (2.3), we get

$$
c_{3}=\frac{\Gamma\left(2-\delta_{1}\right)}{\eta_{1}^{1-\delta_{1}}} \mathcal{J}_{0_{+}}^{\alpha_{1}-\delta_{1}}\left(\phi_{\mathrm{q}}\left(\mathcal{J}_{0_{+}}^{\beta_{1}} \psi\left(\eta_{1}\right)\right)\right) .
$$

Now using condition $v(1)=\frac{\Gamma\left(2-\delta_{1}\right)}{\eta_{1}^{1-\delta_{1}}} \mathcal{J}_{0_{+}}^{\alpha_{1}-\delta_{1}}\left(\phi_{\mathfrak{p}}\left(\mathcal{J}_{0_{+}}^{\beta_{1}} \psi\left(\eta_{1}\right)\right)\right)$ in (2.3), we have

$$
c_{2}=\frac{1}{\Gamma\left(\alpha_{1}\right)} \int_{0}^{1}(1-s)^{\alpha_{1}-1} \phi_{q}\left(\mathcal{J}_{0_{+}}^{\beta_{1}} \psi(s)\right) d s .
$$

By substituting the values of $c_{2}, c_{3}$ in (2.3), we get

$$
\begin{aligned}
v(t)= & {\left[-\frac{1}{\Gamma\left(\alpha_{1}\right)} \int_{0}^{t}(t-s)^{\alpha_{1}-1}+\frac{1}{\Gamma \alpha_{1}} \int_{0}^{1}(1-s)^{\alpha_{1}-1}\right.} \\
& \left.+t \frac{\Gamma\left(2-\delta_{1}\right)}{\eta_{1}^{1-\delta_{1}}} \frac{1}{\Gamma\left(\alpha_{1}-\delta_{1}\right)} \int_{0}^{\eta_{1}}\left(\eta_{1}-s\right)^{\alpha_{1}-\delta_{1}-1}\right] \phi_{\mathrm{q}}\left(\mathcal{J}_{0_{+}}^{\beta_{1}} \psi(\tau)\right) \mathrm{d} s=\int_{0}^{1} \mathcal{G}_{\alpha_{1}}(\mathrm{t}, \mathrm{s}) \phi_{\mathrm{q}}\left(\mathcal{J}_{0_{+}}^{\beta_{1}} \psi(\tau)\right) \mathrm{d} s .
\end{aligned}
$$

With the help of Theorem 2.1, system (1.1) is equivalent to the following coupled system of Hammerstein-type integral equations

$$
\left\{\begin{array}{l}
v(t)=\int_{0}^{1} \mathcal{G}_{\alpha_{1}}(t, s) \phi_{\mathrm{q}}\left(\mathcal{J}_{0_{+}}^{\beta_{1}} \psi_{1}(\tau, z(\tau))\right) d s \\
z(t)=\int_{0}^{1} \mathcal{G}_{\alpha_{2}}(t, s) \phi_{\mathrm{q}}\left(\mathcal{J}_{0_{+}}^{\beta_{2}} \psi_{2}(\tau, v(\tau))\right) d s
\end{array}\right.
$$


where $\mathcal{G}_{\alpha_{2}}(t, s)$ is Green function defined by

$$
\mathcal{G}_{\alpha_{2}}(t, s)= \begin{cases}\frac{-(\mathrm{t}-\mathrm{s})^{\alpha_{2}-1}+(1-s)^{\alpha_{2}-1}}{\Gamma\left(\alpha_{2}\right)}+\frac{\mathrm{t} \Gamma\left(2-\delta_{2}\right)}{\eta_{2}^{1-\delta_{2}} \Gamma\left(\alpha_{2}-\delta_{2}\right)}\left(\eta_{2}-s\right)^{\alpha_{2}-\delta_{2}-1}, & s \leqslant \eta_{2} \leqslant t \leqslant 1, \\ -\frac{(t-s)^{\alpha_{2}-1}}{\Gamma \alpha_{2}}+\frac{(1-s)^{\alpha_{2}-1}}{\Gamma\left(\alpha_{2}\right)}, & \eta_{2} \leqslant s \leqslant t \leqslant 1, \\ \frac{(1-s)^{\alpha_{2}-1}}{\Gamma\left(\alpha_{2}\right)}, & \eta_{2} \leqslant t \leqslant s \leqslant 1 .\end{cases}
$$

From the definitions of $\mathcal{G}_{\alpha_{1}}(t, s)$ and $\mathcal{G}_{\alpha_{2}}(t, s)$, we have

$$
\max _{t \in J}\left|\mathcal{G}_{\alpha_{1}}(t, s)\right|=\frac{(1-s)^{\alpha_{1}-1}}{\Gamma\left(\alpha_{1}\right)}, \quad \max _{t \in J}\left|\mathcal{G}_{\alpha_{2}}(t, s)\right|=\frac{(1-s)^{\alpha_{2}-1}}{\Gamma\left(\alpha_{2}\right)} .
$$

Define operators $\digamma_{1}, \digamma_{2}: \mathcal{U} \rightarrow \mathcal{U}$ as

$$
\digamma_{1}(v(t))=\int_{0}^{1} \mathcal{G}_{\alpha_{1}}(t, s) \phi_{\mathrm{q}}\left(\mathcal{J}_{0_{+}}^{\beta_{1}} \psi_{1}(\tau, z(\tau))\right) \mathrm{d} s, \quad \digamma_{2}(z(\mathrm{t}))=\int_{0}^{1} \mathcal{G}_{\alpha_{2}}(\mathrm{t}, \mathrm{s}) \phi_{\mathrm{q}}\left(\mathcal{J}_{0_{+}}^{\beta_{2}} \psi_{2}(\tau, v(\tau))\right) \mathrm{d} s .
$$

By Theorem 2.1, the solution of coupled system of Hammerstein-type integral equations (2.5) is equivalent to the fixed point, say $(v, z)$, of the operator equation

$$
(v, z)=\digamma(v, z)=\left(\digamma_{1}(v), \digamma_{2}(z)\right) .
$$

Theorem 2.2. With assumption $\left(\mathrm{A}_{1}\right)$, the operator $\digamma: \omega \rightarrow \omega$ is continuous and satisfies the following growth condition

$$
\|\digamma(v, z)\| \leqslant \rho\|(v, z)\|^{p}+\rho^{*},
$$

where $\|(v, z)\|^{p}=\|v\|^{p}+\|z\|^{p}, \rho=\Lambda(a+b), \rho^{*}=\Lambda\left(\mathcal{M}_{1}^{*}+\mathcal{M}_{2}^{*}\right), \Lambda=\max \left\{\frac{(q-1) m^{q-2}}{\Gamma\left(\beta_{1}+1\right)}\left(\frac{2}{\Gamma\left(\alpha_{1}+1\right)}+\right.\right.$ $\left.\left.\eta_{1}^{\alpha_{1}-1} \frac{\Gamma\left(2-\delta_{1}\right)}{\Gamma\left(\alpha_{1}-\delta_{1}+1\right)}\right), \frac{(q-1) m^{q-2}}{\Gamma\left(\beta_{2}+1\right)}\left(\frac{2}{\Gamma\left(\alpha_{2}+1\right)}+\eta_{1}^{\alpha_{2}-1} \frac{\Gamma\left(2-\delta_{2}\right)}{\Gamma\left(\alpha_{2}-\delta_{2}+1\right)}\right)\right\}$, for each $(v, z) \in \omega$.

Proof. Consider a bounded set $\wp_{r}=\{(v, z) \in \omega:\|(v, z)\| \leqslant r\}$ with a sequence $\left\{\left(u_{n}, v_{n}\right)\right\}$ converging to $(v, z)$ in $\wp_{r}$. To show that $\left\|\digamma\left(u_{n}, v_{n}\right)-\digamma(v, z)\right\| \rightarrow 0$ as $n \rightarrow \infty$, we have

$$
\begin{aligned}
& \left|\left(\digamma_{1}\left(v_{\mathrm{n}}\right)-\digamma_{1}(v)\right) \mathrm{t}\right| \\
& \leqslant \mid-\frac{1}{\Gamma\left(\alpha_{1}\right)} \int_{0}^{\mathrm{t}}(\mathrm{t}-\mathrm{s})^{\alpha_{1}-1}+\frac{1}{\Gamma\left(\alpha_{1}\right)} \int_{0}^{1}(1-s)^{\alpha_{1}-1} \\
& \quad+\mathrm{t} \frac{\Gamma\left(2-\delta_{1}\right)}{\eta^{1-\delta_{1}}} \frac{1}{\Gamma\left(\alpha_{1}-\delta_{1}\right)} \int_{0}^{\eta_{1}}\left(\eta_{1}-s\right)^{\alpha_{1}-\delta_{1}-1}|\times| \phi_{\mathrm{q}}\left(\mathcal{J}_{0_{+}}^{\beta_{1}} \psi_{1}\left(\tau, z_{\mathfrak{n}}(\tau)\right)\right)-\phi_{\mathrm{q}}\left(\mathcal{J}_{0_{+}}^{\beta} \psi_{1}(\tau, z(\tau))\right) \mid \mathrm{d} s \\
& \leqslant \\
& \quad(\mathrm{q}-1) \mathrm{m}^{\mathrm{q}-2} \mid-\frac{1}{\Gamma\left(\alpha_{1}\right)} \int_{0}^{\mathrm{t}}(\mathrm{t}-\mathrm{s})^{\alpha_{1}-1}+\frac{1}{\Gamma\left(\alpha_{1}\right)} \int_{0}^{1}(1-s)^{\alpha_{1}-1} \\
& \quad+\mathrm{t} \frac{\Gamma\left(2-\delta_{1}\right)}{\eta_{1}^{1-\delta_{1}}} \frac{1}{\Gamma\left(\alpha_{1}-\delta_{1}\right)} \int_{0}^{\eta_{1}}\left(\eta_{1}-s\right)^{\alpha_{1}-\delta_{1}-1}|\times| \mathcal{J}_{0_{+}}^{\beta_{1}} \psi_{1}\left(\tau, z_{\mathfrak{n}}(\tau)\right)-\mathcal{J}_{0_{+}}^{\beta_{1}} \psi_{1}(\tau, z(\tau)) \mid \mathrm{d} s,
\end{aligned}
$$

and

$$
\begin{aligned}
& \left|\left(\digamma_{2}\left(z_{\mathfrak{n}}\right)-\digamma_{2}(z)\right) \mathrm{t}\right| \\
& \leqslant \mid-\frac{1}{\Gamma\left(\alpha_{2}\right)} \int_{0}^{\mathrm{t}} \frac{(\mathrm{t}-\mathrm{s})^{\alpha_{2}-1}}{\Gamma\left(\alpha_{2}\right)}+\int_{0}^{1} \frac{(1-s)^{\alpha_{2}-1}}{\Gamma\left(\alpha_{2}\right)} \\
& \quad+\mathrm{t} \frac{\Gamma\left(2-\delta_{2}\right)}{\eta_{2}^{1-\delta_{2}}} \frac{1}{\Gamma\left(\alpha_{2}-\delta_{2}\right)} \int_{0}^{\eta_{2}}\left(\eta_{2}-s\right)^{\alpha_{2}-\delta_{2}-1}|\times| \phi_{\mathrm{q}}\left(\mathcal{J}_{0_{+}}^{\beta_{2}} \psi_{2}\left(\tau, \nu_{\mathfrak{n}}(\tau)\right)\right)-\phi_{\mathrm{q}}\left(\mathcal{J}_{0_{+}}^{\beta_{2}} \psi_{2}(\tau, v(\tau))\right) \mid \mathrm{d} s \\
& \leqslant \\
& \quad(\mathrm{q}-1) \mathrm{m}^{\mathrm{q}-2} \mid-\int_{0}^{\mathrm{t}} \frac{(\mathrm{t}-s)^{\alpha_{2}-1}}{\Gamma\left(\alpha_{2}\right)}+\int_{0}^{1} \frac{(1-s)^{\alpha_{2}-1}}{\Gamma\left(\alpha_{2}\right)} \\
& \quad+\mathrm{t} \frac{\Gamma\left(2-\delta_{2}\right)}{\eta_{2}^{1-\delta}} \frac{1}{\Gamma\left(\alpha_{2}-\delta_{2}\right)} \int_{0}^{\eta_{2}}\left(\eta_{2}-s\right)^{\alpha_{2}-\delta_{2}-1}|\times| \mathcal{J}_{0_{+}}^{\beta_{2}} \psi_{2}\left(\tau, \nu_{\mathfrak{n}}(\tau)\right)-\mathcal{J}_{0_{+}}^{\beta_{2}} \psi_{2}(\tau, v(\tau)) \mid \mathrm{d} s .
\end{aligned}
$$


With the help of (2.7) and (2.8), we get

$$
\begin{aligned}
& \left|\left(\digamma\left(v_{\mathrm{n}}, z_{\mathrm{n}}\right)-\digamma(v, z)\right) \mathrm{t}\right| \\
& \leqslant(\mathrm{q}-1) \mathrm{m}^{\mathrm{q}-2} \mid-\int_{0}^{\mathrm{t}} \frac{(\mathrm{t}-\mathrm{s})^{\alpha_{1}-1}}{\Gamma\left(\alpha_{1}\right)}+\int_{0}^{1} \frac{(1-s)^{\alpha_{1}-1}}{\Gamma\left(\alpha_{1}\right)} \\
& \quad+\mathrm{t} \frac{\Gamma\left(2-\delta_{1}\right)}{\eta_{1}^{1-\delta_{1}}} \frac{1}{\Gamma\left(\alpha_{1}-\delta_{1}\right)} \int_{0}^{\eta_{1}}\left(\eta_{1}-s\right)^{\alpha_{1}-\delta_{1}-1}|\times| \mathcal{J}_{0_{+}}^{\beta_{1}} \psi_{1}\left(\tau, z_{\mathrm{n}}(\tau)\right)-\mathcal{J}_{0_{+}}^{\beta_{1}} \psi_{1}(\tau, z(\tau)) \mid \mathrm{d} s \\
& \quad+(\mathrm{q}-1) \mathrm{m}^{\mathrm{q}-2} \mid-\int_{0}^{\mathrm{t}} \frac{(\mathrm{t}-\mathrm{s})^{\alpha_{2}-1}}{\Gamma\left(\alpha_{2}\right)}+\int_{0}^{1} \frac{(1-s)^{\alpha_{2}-1}}{\Gamma\left(\alpha_{2}\right)} \\
& \quad+\mathrm{t} \frac{\Gamma\left(2-\delta_{2}\right)}{\eta_{2}^{1-\delta}} \frac{1}{\Gamma\left(\alpha_{2}-\delta_{2}\right)} \int_{0}^{\eta_{2}}\left(\eta_{2}-s\right)^{\alpha_{2}-\delta_{2}-1}|\times| \mathcal{J}_{0_{+}}^{\beta_{2}} \psi_{2}\left(\tau, \nu_{n}(\tau)\right)-\mathcal{J}_{0_{+}}^{\beta_{2}} \psi_{2}(\tau, v(\tau)) \mid \mathrm{d} s .
\end{aligned}
$$

From the continuity of $\psi_{1}, \psi_{1}$ we have $\psi_{1}\left(\tau, z_{n}(\tau)\right) \rightarrow \psi_{1}(\tau, z(\tau)), \psi_{2}\left(\tau, v_{n}(\tau)\right) \rightarrow \psi_{2}(\tau, v(\tau))$ as $n \rightarrow \infty$. This implies $\left|\mathcal{J}_{0_{+}}^{\beta_{1}} \psi_{1}\left(\tau, z_{\mathrm{n}}(\tau)\right)-\mathcal{J}_{0_{+}}^{\beta_{1}} \psi_{1}(\tau, z(\tau))\right| \rightarrow 0,\left|\mathcal{J}_{0_{+}}^{\beta_{2}} \psi_{2}\left(\tau, \nu_{n}(\tau)\right)-\mathcal{J}_{0_{+}}^{\beta_{2}} \psi_{1}(\tau, v(\tau))\right| \rightarrow 0$ as $\mathrm{n} \rightarrow \infty$. Thus, the operator $\digamma(F)$ is continuous. Now for (2.6), we have

$$
\begin{aligned}
\left|\digamma_{1}(v(t))\right| & =\left|\int_{0}^{1} \mathcal{G}_{\alpha_{1}}(\mathrm{t}, \mathrm{s}) \phi_{\mathrm{q}}\left(\mathcal{J}_{0_{+}}^{\beta_{1}} \psi_{1}(\tau, z(\tau))\right) \mathrm{ds}\right| \\
& \leqslant(\mathrm{q}-1) \mathrm{m}^{\mathrm{q}-2}\left|\int_{0}^{1} \mathcal{G}_{\alpha_{1}}(\mathrm{t}, \mathrm{s})\left(\mathcal{J}_{0_{+}}^{\beta_{1}} \psi_{1}(\tau, z(\tau))\right) \mathrm{d} s\right| \\
& \leqslant \frac{(\mathrm{q}-1) \mathrm{m}^{\mathrm{q}-2}}{\Gamma\left(\beta_{1}+1\right)}\left[\frac{2}{\Gamma\left(\alpha_{1}+1\right)}+\eta_{1}^{\alpha_{1}-1} \frac{\Gamma\left(2-\delta_{1}\right)}{\Gamma\left(\alpha_{1}-\delta_{1}+1\right)}\right]\left(\mathrm{a}\|z\|^{p}+\mathcal{M}_{1}^{*}\right), \\
\left|\digamma_{2}(z(\mathrm{t}))\right| & =\left|\int_{0}^{1} \mathcal{G}_{\alpha_{2}}(\mathrm{t}, \mathrm{s}) \phi_{\mathrm{q}}\left(\mathcal{J}_{0_{+}}^{\beta_{2}} \psi_{2}(\tau, v(\tau))\right) \mathrm{ds}\right| \\
& \leqslant(\mathrm{q}-1) \mathrm{m}^{\mathrm{q}-2} \mid \int_{0}^{1} \mathcal{G}_{\alpha_{2}}(\mathrm{t}, \mathrm{s})\left(\mathcal{J}_{0_{+}}^{\beta_{2}} \psi_{2}(\tau, v(\tau)) \mathrm{d} s \mid\right. \\
& \leqslant \frac{(\mathrm{q}-1) \mathrm{m}^{\mathrm{q}-2}}{\Gamma\left(\beta_{2}+1\right)}\left[\frac{2}{\Gamma\left(\alpha_{2}+1\right)}+\eta_{1}^{\alpha_{2}-1} \frac{\Gamma\left(2-\delta_{2}\right)}{\Gamma\left(\alpha_{2}-\delta_{2}+1\right)}\right]\left(\mathrm{b}\|v\|^{p}+\mathcal{M}_{2}^{*}\right) .
\end{aligned}
$$

With the help of (2.9) and (2.10), we get

$$
\begin{aligned}
\|\digamma(v, z)\|=\left\|\digamma_{1}(v)\right\|+\left\|\digamma_{2}(z)\right\| & \leqslant \Lambda\left(a\|z\|^{p}+\mathcal{M}_{1}^{*}\right)+\Lambda\left(b\|v\|^{p}+\mathcal{M}_{2}^{*}\right) \\
& \leqslant \Lambda(a+b)\left(\|z\|^{p}+\|v\|^{p}\right)+\Lambda\left(\mathcal{M}_{1}^{*}+\mathcal{M}_{2}^{*}\right)=\rho\|(v, z)\|^{p}+\rho^{*}
\end{aligned}
$$

Theorem 2.3. With the assumption $\left(\mathrm{A}_{1}\right)$, the operator $\digamma: \omega \rightarrow \omega$ is compact and $\xi$-Lipschitz with constant zero.

Proof. With the assumption $\left(\mathrm{A}_{1}\right)$, Theorem 2.2 implies that the operator $\digamma$ is bounded. Further, using Lemma 1.10, equation (2.5), and assumption $\left(\mathrm{A}_{1}\right)$, for any $t_{1}, t_{2} \in[0,1]$, we have

$$
\begin{aligned}
\left|\digamma_{1}\left(v\left(t_{1}\right)\right)-\digamma_{1}\left(v\left(t_{2}\right)\right)\right| & =\left|\int_{0}^{1} \mathcal{G}_{\alpha_{1}}\left(t_{1}, s\right) \phi_{\mathrm{q}}\left(\mathcal{J}_{0_{+}}^{\beta_{1}} \psi_{1}(\tau, z(\tau))\right) d s-\int_{0}^{1} \mathcal{G}_{\alpha_{1}}\left(\mathrm{t}_{2}, \mathrm{~s}\right) \phi_{\mathrm{q}}\left(\mathcal{J}_{0_{+}}^{\beta_{1}} \psi_{1}(\tau, z(\tau))\right) \mathrm{d} s\right| \\
& \leqslant(\mathrm{q}-1) \mathrm{m}^{\mathrm{q}-2}\left|\int_{0}^{1} \mathcal{G}_{1}\left(\mathrm{t}_{1}, \mathrm{~s}\right)-\int_{0}^{1} \mathcal{G}_{1}\left(\mathrm{t}_{2}, \mathrm{~s}\right)\right| \mathcal{J}_{0_{+}}^{\beta_{1}} \psi_{1}(\tau, z(\tau)) \mid \mathrm{d} s \\
& \leqslant \frac{(\mathrm{q}-1) \mathrm{m}^{\mathrm{q}-2}}{\Gamma\left(\beta_{1}+1\right)} \mid\left(\mathrm{t}_{1}-\mathrm{t}_{2}\right) \frac{\Gamma\left(2-\delta_{1}\right)}{\eta_{1}^{1-\delta_{1}}} \frac{1}{\Gamma\left(\alpha_{1}-\delta_{)}\right.} \int_{0}^{\eta_{1}}\left(\eta_{1}-\mathrm{s}\right)^{\alpha_{1}-\delta_{1}-1} \mathrm{~d} s
\end{aligned}
$$




$$
-\frac{1}{\Gamma \alpha_{1}}\left(\int_{0}^{t_{1}}\left(t_{1}-s\right)^{\alpha_{1}-1} d s-\int_{0}^{t_{2}}\left(t_{2}-s\right)^{\alpha_{1}-1} d s\right) \mid\left(a\|z\|^{p}+\mathcal{M}_{1}^{*}\right)
$$

By simplification, we have

$$
\left|\digamma_{1}\left(v\left(t_{1}\right)\right)-\digamma_{1}\left(v\left(t_{2}\right)\right)\right| \leqslant \frac{(q-1) m^{q-2}}{\Gamma\left(\beta_{1}+1\right)}\left[\eta_{1}^{\alpha_{1}-1} \frac{\Gamma\left(2-\delta_{1}\right)\left|t_{1}-t_{2}\right|}{\Gamma\left(\alpha_{1}-\delta_{1}+1\right)}+\frac{\left|t_{1}^{\alpha_{1}}-t_{2}^{\alpha_{1}}\right|}{\Gamma\left(\alpha_{1}+1\right)}\right]\left(a\|z\|^{p}+\mathcal{M}_{1}^{*}\right) .
$$

Similarly, we have

$$
\left|\digamma_{2}\left(z\left(t_{1}\right)\right)-\digamma_{2}\left(z\left(t_{2}\right)\right)\right| \leqslant \frac{(q-1) m^{q-2}}{\Gamma\left(\beta_{2}+1\right)}\left[\eta_{2}^{\alpha_{2}-1} \frac{\Gamma\left(2-\delta_{2}\right)\left|t_{1}-t_{2}\right|}{\Gamma\left(\beta_{2}-\delta_{2}+1\right)}+\frac{\left|t_{1}^{\alpha_{2}}-t_{2}^{\alpha_{2}}\right|}{\Gamma\left(\alpha_{2}+1\right)}\right]\left(b\|v\|^{p}+\mathcal{M}_{2}^{*}\right) .
$$

From (2.11) and (2.12) we have

$$
\begin{aligned}
\left|\digamma(v, z)\left(t_{1}\right)-\digamma(v, z)\left(t_{2}\right)\right| \leqslant & \frac{(q-1) m^{q-2}}{\Gamma\left(\beta_{1}+1\right)}\left[\eta_{1}^{\alpha_{1}-1} \frac{\Gamma\left(2-\delta_{1}\right)\left|t_{1}-t_{2}\right|}{\Gamma\left(\alpha_{1}-\delta_{1}+1\right)}+\frac{\left|t_{1}^{\alpha_{1}}-t_{2}^{\alpha_{1}}\right|}{\Gamma\left(\alpha_{1}+1\right)}\right]\left(a\|z\|^{p}+\mathcal{M}_{1}^{*}\right) \\
& +\frac{(q-1) m^{q-2}}{\Gamma\left(\beta_{2}+1\right)}\left[\eta_{2}^{\alpha_{2}-1} \frac{\Gamma\left(2-\delta_{2}\right)\left|t_{1}-t_{2}\right|}{\Gamma\left(\beta_{2}-\delta_{2}+1\right)}+\frac{\left|t_{1}^{\alpha_{2}}-t_{2}^{\alpha_{2}}\right|}{\Gamma\left(\alpha_{2}+1\right)}\right]\left(b\|v\|^{p}+\mathcal{M}_{2}^{*}\right) .
\end{aligned}
$$

Equation (2.13) tends to zero as $t_{1} \rightarrow t_{2}$. Thus $\digamma=\left(\digamma_{1}, \digamma_{2}\right)$ is equicontinuous on D. Consequently, by Arzelà-Ascoli theorem $\digamma(D)$ is compact. Hence $\mathrm{D}$ is $\xi$-Lipschitz with constant zero.

Theorem 2.4. Assume that $\left(\mathrm{A}_{1}\right)$ and $\left(\mathrm{A}_{2}\right)$ hold. Then the coupled system (1.1) has a unique solution provided that $\Delta^{*}<1$, where

$$
\begin{aligned}
\Delta^{*}= & \frac{(q-1) m^{q-2} L_{\psi_{1}}}{\Gamma\left(\beta_{1}+1\right)}\left[\frac{2}{\Gamma\left(\alpha_{1}+1\right)}+\eta_{1}^{\alpha_{1}-1} \frac{\Gamma\left(2-\delta_{1}\right)}{\Gamma\left(\alpha_{1}-\delta_{1}+1\right)}\right] \\
& +\frac{(q-1) m^{q-2} L_{\psi_{2}}}{\Gamma\left(\beta_{2}+1\right)}\left[\frac{2}{\Gamma\left(\alpha_{2}+1\right)}+\eta_{2}^{\alpha_{2}-1} \frac{\Gamma\left(2-\delta_{2}\right)}{\Gamma\left(\alpha_{2}-\delta_{2}+1\right)}\right] .
\end{aligned}
$$

Proof. Suppose that $(v, z),(\bar{v}, \bar{z}) \in \omega$ are two different solutions of the coupled system of FDEs with p-Laplacian operator (1.1). With the help of Lemma 1.10, (2.5), and assumption $\left(\mathrm{A}_{1}\right)$, we have

$$
\begin{aligned}
\left|\digamma_{1}(v(t))-\digamma_{1}(\bar{v}(t))\right| & =\left|\int_{0}^{1} \mathcal{G}_{\alpha_{1}}(\mathrm{t}, \mathrm{s}) \phi_{\mathrm{q}}\left(\mathcal{J}_{0_{+}}^{\beta_{1}} \psi_{1}(\tau, \bar{z}(\tau))\right) \mathrm{d} s-\int_{0}^{1} \mathcal{G}_{\alpha_{1}}(\mathrm{t}, \mathrm{s}) \phi_{\mathrm{q}}\left(\mathcal{J}_{0_{+}}^{\beta_{1}} \psi_{1}(\tau, \bar{z}(\tau))\right) \mathrm{d} s\right| \\
& \leqslant \frac{(\mathrm{q}-1) \mathrm{m}^{\mathrm{q}-2} \mathrm{~L}_{\psi_{1}}}{\Gamma\left(\beta_{1}+1\right)}\left[\frac{2}{\Gamma\left(\alpha_{1}+1\right)}+\eta_{1}^{\alpha_{1}-1} \frac{\Gamma\left(2-\delta_{1}\right)}{\Gamma\left(\alpha_{1}-\delta_{1}+1\right)}\right]|z(\mathrm{t})-\bar{z}(\mathrm{t})|, \\
\left|\digamma_{2}(z(\mathrm{t}))-\digamma_{2}(\bar{z}(\mathrm{t}))\right| & =\left|\int_{0}^{1} \mathcal{G}_{\alpha_{2}}(\mathrm{t}, \mathrm{s}) \phi_{\mathrm{q}}\left(\mathcal{J}_{0_{+}}^{\beta_{2}} \psi_{2}(\tau, \bar{v}(\tau))\right) \mathrm{d} s-\int_{0}^{1} \mathcal{G}_{\alpha_{2}}(\mathrm{t}, \mathrm{s}) \phi_{\mathrm{q}}\left(\mathcal{J}_{0_{+}}^{\beta_{2}} \psi_{2}(\tau, \bar{v}(\tau))\right) \mathrm{d} s\right| \\
& \leqslant \frac{(\mathrm{q}-1) \mathrm{m}^{\mathrm{q}-2} \mathrm{~L}_{\psi_{2}}}{\Gamma\left(\beta_{2}+1\right)}\left[\frac{2}{\Gamma\left(\alpha_{2}+1\right)}+\eta_{1}^{\alpha_{2}-1} \frac{\Gamma\left(2-\delta_{2}\right)}{\Gamma\left(\alpha_{2}-\delta_{2}+1\right)}\right]|v(\mathrm{t})-\bar{v}(\mathrm{t})| .
\end{aligned}
$$

From (2.14) and (2.15), we have

$$
\begin{aligned}
|\digamma(v, z)(t)-\digamma(\bar{v}, \bar{z})(t)| \leqslant & \frac{(q-1) m^{q-2} L_{\psi_{1}}}{\Gamma\left(\beta_{1}+1\right)}\left[\frac{2}{\Gamma\left(\alpha_{1}+1\right)}+\eta_{1}^{\alpha_{1}-1} \frac{\Gamma\left(2-\delta_{1}\right)}{\Gamma\left(\alpha_{1}-\delta_{1}+1\right)}\right]|z(t)-\bar{z}(t)| \\
& +\frac{(q-1) m^{q-2} L_{\psi_{2}}}{\Gamma\left(\beta_{2}+1\right)}\left[\frac{2}{\Gamma\left(\alpha_{2}+1\right)}+\eta_{2}^{\alpha_{2}-1} \frac{\Gamma\left(2-\delta_{2}\right)}{\Gamma\left(\alpha_{2}-\delta_{2}+1\right)}\right]|v(t)-\bar{v}(t)| \\
\leqslant & \frac{(q-1) m^{q-2} L_{\psi_{1}}}{\Gamma\left(\beta_{1}+1\right)}\left[\frac{2}{\Gamma\left(\alpha_{1}+1\right)}+\eta_{1}^{\alpha_{1}-1} \frac{\Gamma\left(2-\delta_{1}\right)}{\Gamma\left(\alpha_{1}-\delta_{1}+1\right)}\right] \\
& +\frac{(q-1) m^{q-2} L_{\psi_{2}}}{\Gamma\left(\beta_{2}+1\right)}\left[\frac{2}{\Gamma\left(\alpha_{2}+1\right)}+\eta_{2}^{\alpha_{2}-1} \frac{\Gamma\left(2-\delta_{2}\right)}{\Gamma\left(\alpha_{2}-\delta_{2}+1\right)}\right] \\
& \times(|v(t)-\bar{v}(t)|+|z(t)-\bar{z}(t)|) .
\end{aligned}
$$


By the help of Banach's fixed point theorem $\digamma$ has a unique fixed point. Consequently, the coupled system of FDEs with p-Laplacian operator (1.1) has a unique solution.

\section{Hyers-Ulam stability}

In this section, we study Hyers-Ulam stability for the coupled system of FDEs with fractional differential and integral initial and boundary conditions with p-Laplacian operator (1.1).

Definition 3.1. The coupled system of Hammerstein-type integral equations (2.4) is Hyres-Ulam stable if there exist positive constants $\mathcal{D}_{1}, \mathcal{D}_{2}$ satisfying: for every $\lambda_{1}, \lambda_{2}>0$, if

$$
\left|v(t)-\int_{0}^{1} \mathcal{G}_{\alpha_{1}}(t, s) \phi_{\mathrm{q}}\left(\mathcal{J}_{0_{+}}^{\beta_{1}} \psi_{1}(\tau, z(\tau))\right) d s\right| \leqslant \lambda_{1}, \quad\left|z(t)-\int_{0}^{1} \mathcal{G}_{\alpha_{2}}(t, s) \phi_{q}\left(\mathcal{J}_{0_{+}}^{\beta_{2}} \psi_{2}(\tau, v(\tau))\right) d s\right| \leqslant \lambda_{2}
$$

there exists a pair, say $\left(v^{*}(t), z^{*}(t)\right)$, satisfying

$$
v^{*}(t)=\int_{0}^{1} \mathcal{G}_{\alpha_{1}}(t, s) \phi_{\mathrm{q}}\left(\mathcal{J}_{0_{+}}^{\beta_{1}} \psi_{1}\left(s, z^{*}(s)\right)\right) d s, \quad z^{*}(t)=\int_{0}^{1} \mathcal{G}_{\alpha_{2}}(t, s) \phi_{\mathrm{q}}\left(\mathcal{J}_{0_{+}}^{\beta_{2}} \psi_{2}\left(\tau, v^{*}(\tau)\right)\right) d s,
$$

such that

$$
\left|v(t)-v^{*}(t)\right| \leqslant \mathcal{D}_{1} \lambda_{1}, \quad\left|z(t)-z^{*}(t)\right| \leqslant \mathcal{D}_{2} \lambda_{2}
$$

Theorem 3.2. With assumptions $\left(\mathrm{A}_{1}\right)$ and $\left(\mathrm{A}_{2}\right)$, the coupled system of FDEs with p-Laplacian operator (1.1) is Hyers-Ulam stable.

Proof. With the help of Theorem 2.4, let $(v(t), z(t))$ be unique solution of the coupled system of Hammerstein-type integral equations (2.4). Let $\left(v^{*}(t), z^{*}(t)\right)$ be any other approximation satisfying (3.1). Then, by the help of Definition 3.1 we have

$$
\begin{aligned}
\left|v(t)-v^{*}(\mathrm{t})\right| & \leqslant \mid \int_{0}^{1} \digamma \alpha_{1}(\mathrm{t}, \mathrm{s})\left(\phi_{\mathrm{q}}\left(\mathcal{J}_{0_{+}}^{\beta_{1}} \psi_{1}(\tau, z(\tau))-\phi_{\mathrm{q}}\left(\mathcal{J}_{0_{+}}^{\beta_{1}} \psi_{1}\left(\tau, z^{*}(\tau)\right)\right)\right) \mathrm{d} s \mid\right. \\
& \leqslant\left[\frac{2}{\Gamma\left(\alpha_{1}+1\right)}+\eta_{1}^{\alpha_{1}-1} \frac{\Gamma\left(2-\delta_{1}\right)}{\Gamma\left(\alpha_{1}-\delta_{1}+1\right)}\right]\left|\phi_{\mathrm{q}}\left(\mathcal{J}_{0_{+}}^{\beta_{1}} \psi_{1}(\tau, z(\tau))\right)-\phi_{\mathrm{q}}\left(\mathcal{J}_{0_{+}}^{\beta_{1}} \psi_{1}\left(\tau, z^{*}(\tau)\right)\right)\right| \\
& \leqslant \frac{(\mathrm{q}-1) \mathrm{m}^{\mathrm{q}-2}}{\Gamma\left(\beta_{1}+1\right)} \mathrm{L}_{\psi_{1}}\left[\frac{2}{\Gamma\left(\alpha_{1}+1\right)}+\eta_{1}^{\alpha_{1}-1} \frac{\Gamma\left(2-\delta_{1}\right)}{\Gamma\left(\alpha_{1}-\delta_{1}+1\right)}\right]\left|z-z^{*}\right|=\mathcal{D}_{1} \lambda_{1}, \\
\left|z(\mathrm{t})-z^{*}(\mathrm{t})\right| & \leqslant \mid \int_{0}^{1} \digamma_{\alpha_{2}}(\mathrm{t}, \mathrm{s})\left(\phi_{\mathrm{q}}\left(\mathcal{J}_{0_{+}}^{\beta_{2}} \psi_{1}(\tau, v(\tau))-\phi_{\mathrm{q}}\left(\mathcal{J}_{0_{+}}^{\beta_{2}} \psi_{2}\left(\tau, v^{*}(\tau)\right)\right)\right) \mathrm{ds} \mid\right. \\
& \leqslant\left[\frac{2}{\Gamma\left(\alpha_{2}+1\right)}+\eta_{2}^{\alpha_{2}-1} \frac{\Gamma\left(2-\delta_{2}\right)}{\Gamma\left(\alpha_{2}-\delta_{2}+1\right)}\right]\left|\phi_{\mathrm{q}}\left(\mathcal{J}_{0_{+}}^{\beta_{2}} \psi_{1}(\tau, v(\tau))\right)-\phi_{\mathrm{q}}\left(\mathcal{J}_{0_{+}}^{\beta_{2}} \psi_{1}\left(\tau, v^{*}(\tau)\right)\right)\right| \\
& \leqslant \frac{(\mathrm{q}-1) \mathrm{m}^{\mathrm{q}-2}}{\Gamma\left(\beta_{2}+1\right)} \mathrm{L}_{\psi_{2}}\left[\frac{2}{\Gamma\left(\alpha_{2}+1\right)}+\eta_{2}^{\alpha_{2}-1} \frac{\Gamma\left(2-\delta_{2}\right)}{\Gamma\left(\alpha_{2}-\delta_{2}+1\right)}\right]\left|v-v^{*}\right|=\mathcal{D}_{2} \lambda_{2},
\end{aligned}
$$

where

$$
\left\{\begin{array}{l}
\mathcal{D}_{1}=\frac{(q-1) m^{q-2}}{\Gamma\left(\beta_{1}+1\right)} L_{\psi_{1}}\left[\frac{2}{\Gamma\left(\alpha_{1}+1\right)}+\eta_{1}^{\alpha_{1}-1} \frac{\Gamma\left(2-\delta_{1}\right)}{\Gamma\left(\alpha_{1}-\delta_{1}+1\right)}\right] \\
\mathcal{D}_{2}=\frac{(q-1) m^{q-2}}{\Gamma\left(\beta_{2}+1\right)} L_{\psi_{2}}\left[\frac{2}{\Gamma\left(\alpha_{2}+1\right)}+\eta_{2}^{\alpha_{2}-1} \frac{\Gamma\left(2-\delta_{2}\right)}{\Gamma\left(\alpha_{2}-\delta_{2}+1\right)}\right]
\end{array}\right.
$$

Hence with the help of (3.2) and (3.3), the coupled system (2.4) is Hyers-Ulam stable which implies the coupled system of FDEs with p-Laplacian operator (1.1) is Hyers-Ulam stable. 


\section{Illustrative example}

Here we give application of the results proved in Sections 2 and 3.

Example 4.1. Consider the following coupled system of FDEs with p-Laplacian operator and fractional order differential and integral initial and boundary conditions of the type

$$
\begin{aligned}
& \mathfrak{D}_{0_{+}}^{\frac{5}{4}} \phi_{5}\left(\mathfrak{D}_{0_{+}}^{\frac{3}{2}} v(t)\right)=-\psi_{1}(t, z(t)), \quad \mathfrak{D}_{0_{+}}^{\frac{5}{4}} \phi_{5}\left(\mathfrak{D}_{0_{+}}^{\frac{3}{2}} z(t)\right)=-\psi_{2}(t, v(t)), \\
& \mathfrak{D}_{0_{+}}^{\frac{3}{2}} v(0)=0=\left.\phi_{5}\left(\mathfrak{D}_{0_{+}}^{\frac{3}{2}} v(t)\right)^{\prime}\right|_{t=0}=\left.\mathfrak{D}_{0_{+}}^{\frac{4}{5}} v(t)\right|_{t=\frac{1}{2}}, v(1)=\left.\frac{\Gamma\left(2-\frac{4}{5}\right)}{\frac{1}{2}^{\left(1-\frac{4}{5}\right)}} \mathfrak{J}_{0_{+}}^{\left(\frac{3}{2}-\frac{4}{5}\right)} \phi_{\frac{5}{4}}\left(\mathcal{J}_{0_{+}}^{\frac{5}{4}} \psi_{1}(t, z(t))\right)\right|_{t=\frac{1}{2}}, \\
& \mathfrak{D}_{0_{+}}^{\frac{3}{2}} z(0)=0=\left.\phi_{5}\left(\mathfrak{D}_{0_{+}}^{\frac{3}{2}} z(t)\right)^{\prime}\right|_{t=0}=\left.\mathfrak{D}_{0_{+}}^{\frac{4}{5}} z(t)\right|_{t=\frac{1}{2}}, \quad v(1)=\left.\frac{\Gamma\left(2-\frac{4}{5}\right)}{\frac{1}{2}^{\left(1-\frac{4}{5}\right)}} \mathcal{J}_{0_{+}}^{\left(\frac{3}{2}-\frac{4}{5}\right)} \phi_{\frac{5}{4}}\left(\mathcal{J}_{0_{+}}^{\frac{5}{4}} \psi_{2}(t, v(t))\right)\right|_{t=\frac{1}{2}}
\end{aligned}
$$

where $t \in[0,1], p=5, \alpha_{i}=3 / 2, \beta_{i}=5 / 4, \delta_{i}=4 / 5, \eta_{i}=\frac{1}{2}$ for $i=1,2$ and $m=2, L_{\psi_{i}}=1 / 15$ and

$$
\psi_{1}(t, v(t))=\frac{-25}{17}+\frac{1}{15} \cos (v(t)), \quad \psi_{2}(t, z(t))=\frac{30}{18}+\frac{1}{15} \sin (z(t)) .
$$

It is easy to check that

$$
\begin{aligned}
\Delta^{*}= & \frac{(q-1) m^{q-2} L_{\psi_{1}}}{\Gamma\left(\beta_{1}+1\right)}\left[\frac{2}{\Gamma\left(\alpha_{1}+1\right)}+\eta_{1}^{\frac{3}{2}-1} \frac{\Gamma\left(2-\delta_{1}\right)}{\Gamma\left(\alpha_{1}-\delta_{1}+1\right)}\right] \\
& +\frac{(q-1) m^{q-2} L_{\psi_{2}}}{\Gamma\left(\beta_{2}+1\right)}\left[\frac{2}{\Gamma\left(\alpha_{2}+1\right)}+\eta_{2}^{\frac{3}{2}-1} \frac{\Gamma\left(2-\delta_{2}\right)}{\Gamma\left(\alpha_{2}-\delta_{2}+1\right)}\right] \\
= & \frac{\left(\frac{5}{4}-1\right) 2^{\frac{5}{4}-2}}{15 \Gamma\left(\frac{5}{4}+1\right)}\left[\frac{2}{\Gamma\left(\frac{3}{2}+1\right)}+0.5^{\frac{3}{2}-1} \frac{\Gamma\left(2-\frac{4}{5}\right)}{\Gamma\left(\frac{3}{2}-\frac{4}{5}+1\right)}\right]+\frac{\left(\frac{5}{4}-1\right) 2^{\frac{5}{4}-2}}{15 \Gamma\left(\frac{5}{4}+1\right)}\left[\frac{2}{\Gamma\left(\frac{3}{2}+1\right)}+0.5^{\frac{3}{2}-1} \frac{\Gamma\left(2-\frac{4}{5}\right)}{\Gamma\left(\frac{3}{2}-\frac{4}{5}+1\right)}\right]<1 .
\end{aligned}
$$

Therefore, from Theorem 2.4, we conclude that (4.1) has a unique solution. Similarly, the conditions of Theorem 3.2 may be verified. Thus the system (4.1) is Hyers-Ulam stable and has a unique solution.

\section{Conclusion}

In this paper, we have studied the existence and uniqueness of solution and Hyers-Ulam stability for a coupled system of FDEs, with p-Laplacian operator and fractional order differential and integral initial and boundary conditions of the type (1.1). We used topological degree theory, Arzelà-Ascoli, and Banach fixed point theorems for this study. The proofs are very simple and may be carried out in the case of q-difference similarly.

\section{Acknowledgment}

This work was supported by the National Natural Science Foundation of China (11571378) and China Government Excellent Young Talent Program.

\section{References}

[1] A. Ali, B. Samet, K. Shah, R. A. Khan, Existence and stability of solution to a toppled systems of differential equations of non-integer order, Bound. Value Probl., 2017 (2017), 13 pages. 1

[2] G. A. Anastassiou, On right fractional calculus, Chaos Solitons Fractals, 42 (2009), 365-376. 1

[3] D. Baleanu, R. P. Agarwal, H. Mohammadi, S. Rezapour, Some existence results for a nonlinear fractional differential equation on partially ordered Banach spaces, Bound. Value Probl., 2013 (2013), 8 pages. 1 
[4] D. Băleanu, O. G. Mustafa, On the global existence of solutions to a class of fractional differential equations, Comput. Math. Appl., 59 (2010), 1835-1841. 1

[5] D. Băleanu, O. G. Mustafa, R. P. Agarwal, An existence result for a superlinear fractional differential equation, Appl. Math. Lett., 23 (2010), 1129-1132. 1

[6] D. Băleanu, O. G. Mustafa, R. P. Agarwal, On the solution set for a class of sequential fractional differential equations, J. Phys. A, 43 (2010), 7 pages. 1

[7] J. Brzdęk, L. Cădariu, K. Ciepliński, A. Fošner, Z. Leśniak, Survey on recent Ulam stability results concerning derivations, J. Funct. Spaces, 2016 (2016), 9 pages. 1

[8] M. Caputo, Linear models of dissipation whose Q is almost frequency independent, II, Reprinted from Geophys. J. R. Astr. Soc., 13 (1967), 529-539, Fract. Calc. Appl. Anal., 11 (2008), 4-14. 1, 1

[9] L.-L. Cheng, W.-B. Liu, Q.-Q. Ye, Boundary value problem for a coupled system of fractional differential equations with p-Laplacian operator at resonance, Electron. J. Differential Equations, 2014 (2014), 12 pages. 1

[10] P. Găvruţă, S.-M. Jung, Y.-J. Li, Hyers-Ulam stability for second-order linear differential equations with boundary conditions, Electron. J. Differential Equations, 2011 (2011), 5 pages. 1, 1.11

[11] A. Granas, J. Dugundji, Fixed point theory, Springer Monographs in Mathematics, Springer-Verlag, New York, (2003). 1

[12] R. Hilfer (Ed.), Applications of fractional calculus in physics, World Scientific Publishing Co., Inc., River Edge, NJ, (2000). 1

[13] Z.-G. Hu, W.-B. Liu, J.-Y. Liu, Existence of solutions for a coupled system of fractional p-Laplacian equations at resonance, Adv. Difference Equ., 2013 (2013), 14 pages. 1

[14] F. Isaia, On a nonlinear integral equation without compactness, Acta Math. Univ. Comenian. (N.S.), 75 (2006), $233-240$. 1.9

[15] H. Jafari, D. Baleanu, H. Khan, R. A. Khan, A. Khan, Existence criterion for the solutions of fractional order p-Laplacian boundary value problems, Bound. Value Probl., 2015 (2015), 10 pages. 1

[16] R. A. Khan, A. Khan, Existence and uniqueness of solutions for p-Laplacian fractional order boundary value problems, Comput. Methods Differ. Equ., 2 (2014), 205-215.

[17] R. A. Khan, A. Khan, A. Samad, H. Khan, On existence of solutions for fractional differential equation with $p$-Laplacian operator, J. Fract. Calc. Appl., 5 (2014), 28-37. 1

[18] A. A. Kilbas, H. M. Srivastava, J. J. Trujillo, Theory and applications of fractional differential equations, North-Holland Mathematics Studies, Elsevier Science B.V., Amsterdam, (2006) 1, 1.3

[19] P. Kumam, A. Ali, K. Shah, R. A. Khan, Existence results and Hyers-Ulam stability to a class of nonlinear arbitrary order differential equations, J. Nonlinear Sci. Appl., 10 (2017), 2986-2997. 1

[20] N. I. Mahmudov, S. Unul, Existence of solutions of $\alpha \in(2,3]$ order fractional three-point boundary value problems with integral conditions, Abstr. Appl. Anal., 2014 (2014), 12 pages. 1

[21] N. I. Mahmudov, S. Unul, Existence of solutions of fractional boundary value problems with p-Laplacian operator, Bound. Value Probl., 2015 (2015), 16 pages. 1

[22] N. I. Mahmudov, S. Unul, On existence of BVP's for impulsive fractional differential equations, Adv. Difference Equ., 2017 (2017), 16 pages. 1

[23] K. S. Miller, B. Ross, An introduction to the fractional calculus and fractional differential equations, A Wiley-Interscience Publication, John Wiley \& Sons, Inc., New York, (1993). 1

[24] K. B. Oldham, J. Spainer, The fractional calculus, Theory and applications of differentiation and integration to arbitrary order, With an annotated chronological bibliography by Bertram Ross, Mathematics in Science and Engineering, Academic Press [A subsidiary of Harcourt Brace Jovanovich, Publishers], New York-London, (1974).

[25] I. Podlubny, Fractional differential equations, An introduction to fractional derivatives, fractional differential equations, to methods of their solution and some of their applications, Mathematics in Science and Engineering, Academic Press, Inc., San Diego, CA, (1999). 1

[26] K. R. Prasad, B. M. B. Krushna, Multiple positive solutions for a coupled system of $\mathrm{p}$-Laplacian fractional order two-point boundary value problems, Int. J. Differ. Equ., 2014 (2014), 10 pages. 1

[27] S. G. Samko, A. A. Kilbas, O. I. Marichev, Fractional integrals and derivatives, Theory and applications, Edited and with a foreword by S. M. Nikol'skii, Translated from the 1987 Russian original, Revised by the authors, Gordon and Breach Science Publishers, Yverdon, (1993).

[28] T.-F. Shen, W.-B. Liu, X.-H. Shen, Existence and uniqueness of solutions for several BVPs of fractional differential equations with p-Laplacian operator, Mediterr. J. Math., 13 (2016), 4623-4637. 1.10

[29] X.-W. Su, Boundary value problem for a coupled system of nonlinear fractional differential equations, Appl. Math. Lett., 22 (2009), 64-69. 1

[30] H.-G. Sun, Z.-P. Li, Y. Zhang, W. Chen, Fractional and fractal derivative models for transient anomalous diffusion: model comparison, Chaos Solitons Fractals, 102 (2017), 346-353. 1

[31] H.-G. Sun, Y. Zhang, W. Chen, D. M. Reeves, Use of a variable-index fractional-derivative model to capture transient dispersion in heterogeneous media, J. Contam. Hydrol., 157 (2014), 47-58. 1

[32] C. Urs, Coupled fixed point theorems and applications to periodic boundary value problems, Miskolc Math. Notes, 14 (2013), 323-333. 1 